\title{
Effect of charged sand particles on microwave propagation along earth-space paths
}

\author{
Qunfeng Dong, Li Wang, Yingle Li, Mingjun Wang \\ Xianyang Normal College \\ Xianyang, China \\ qunfengdong1028@163.com
}

\author{
Jiadong Xu, Baoping Wang \\ The Electronic Information Institute \\ Northwestern Polytechnical University \\ Xi'an, China
}

\begin{abstract}
According to the forward scattering amplitude function of the charged sand particles under the Rayleigh approximation, the calculation models of attenuation and phase shift for microwave wave through sand storms incorporating charged sand particles are obtained along horizontal paths. The attenuation and phase shift due to sand storms along earth-space paths are also given. Some results with different visibility, moisture content, and charge-to-mass are obtained and discussed.

Index Terms-Microwave, earth-space paths, changed sand particles.
\end{abstract}

\section{INTRODUCTION}

The theory of microwave propagation in sand storms has received much attention in the literature owing to the importance of radio relay, communication and remote sensing. A number of papers have addressed the problem of prediction of the amount of attenuation and phase shift in sand storms [1][5].

Microwave propagation in sand storms along earth-space or slant paths has been investigated by several workers[6]-[9].The performance of satellite systems operating in the $\mathrm{Ku}$ and $\mathrm{Ka}$ bands is more sensitive on the propagation characteristics of the transmission medium. At frequencies above $10 \mathrm{GHz}$, sand storms effect can cause microwave degradation on earth-space paths for substantial percentages of time, which leads to reduction in the quality and availability of communication services [10].

Many measurements and theories show that sand particles carry charges, and the charges influence the saltation of sand particles obviously [11]. For a single spherical sand particle considering part charges on sand particle, using Rayleigh scattering theory, there is the forward scattering amplitude function of charged sand particle [12]. According to the lognormal distribution, microwave attenuation induced by the charged sand is analyzed in the literature [13]. However, considerably less work has been reported on the microwave propagation along earth-space paths in charged sand storms.

The aim of this paper is (i) based on forward propagation constants, to present a general formula of propagation of microwave in sand storms incorporating charged sand particles suitable for equisized particle distribution (ii) To develop a model for the calculation of microwave attenuation and phase shift in Earth-satellite links.

\section{MicrowaVe AtTENUATION AND Phase ShIFT IN SAND STORMS}

Here we follow the analysis of chu [1], and Ansri and Evans [3] which are based on the slab of particles model. This gives rise to forward propagation constants

$$
K_{V, H}(\alpha)=k_{0}+\frac{2 \pi}{k_{0}} \int_{0}^{\infty} f_{V, H}(\alpha, a) N(a) d a
$$

where $k_{0}$ is the propagation constant in free-space, $\varphi$ is the elevation angle of the incident radiation and $\mathrm{N}(\mathrm{r})=\mathrm{N}_{0} \mathrm{P}(\mathrm{r})$ is the particle size distribution per unit volume $\left(\mathrm{cm}^{3}\right)$ having radii in the region $r \rightarrow r+d r, f_{V, H}(\varphi, r)$ is the forward scattering amplitude for vertical and horizontal polarizations, as indicated by the subscripts $\mathrm{V}$ and $\mathrm{H}$.

The attenuation and phase shift can then be determined directly as

$$
\begin{array}{ll}
\alpha=8.686 \operatorname{Im}\left[K_{V, H}\right] & {[\mathrm{dB} / \mathrm{m}]} \\
\beta=\frac{180}{\pi} \operatorname{Re}\left[K_{V, H}\right] & {[\mathrm{deg} / \mathrm{m}]}
\end{array}
$$

III. Microwave AtTenuation ANd Phase Shift In SAND STORMS INCORPORATING CHARGED SAND PARTICLES FOR EQUISIZED PARTICLE

As the sand particle size is relatively small and the frequency is not too high, i.e. $k a<<1$, using the Rayleigh approximation, the forward scattering amplitude of the charged sand [12] is

$$
f=k_{0}^{2}\left(\frac{\varepsilon_{m}^{*}-1}{\varepsilon_{m}^{*}+2}\right) a^{3}+k_{0}^{2} a^{3} \frac{a \rho q\left(\varepsilon_{m}^{*}-1\right) \sin ^{2} \theta_{0}}{6 \varepsilon_{0} E_{0}\left(1-\cos \theta_{0}\right)}
$$

where a is the radius of sand particle, $\rho$ is the mass density of sand particle, $q$ is the charge-to-mass ratio,$\theta_{0}$ is the charge distribution angle of a single spherical sand particle and it is assumed that the distribution of charge is in the shape of a spherical cap and the charges distribute uniformly, $E_{0}$ is the intensity of incident electric field and $\varepsilon_{0}$ is the permittivity of vacuum, $\varepsilon_{m}^{*}$ is the dielectric constant of sand particle.

For equisized particle, the propagation constant can be expressed as 


$$
K=k_{0}\left[1+2 \pi a^{3} N\left(\frac{\varepsilon_{m}^{*}-1}{\varepsilon_{m}^{*}+2}\right)+2 \pi a^{4} N \frac{\rho q\left(\varepsilon_{m}^{*}-1\right) \sin ^{2} \theta_{0}}{6 \varepsilon_{0} E_{0}\left(1-\cos \theta_{0}\right)}\right]
$$

where $\mathrm{N}$ is the concentration, i.e. the number of particles per unit volume.

Most meteorological observations of sand and dust storms are made in terms of optical visibility rather than concentration. It is further useful to adopt the optical attenuation coefficient $\alpha_{0}$, which is inversely proportional to visibility [3]

$$
V_{b}=15 / \alpha_{0}
$$

where $\alpha_{0}$ is measured in decibels per kilometer and is related to the concentration via

$$
N=\frac{\alpha_{0} a}{6.5\left(\frac{4 \pi}{3} a^{3}\right)}
$$

Thus, in terms of visibility, the propagation coefficient

$$
K=k_{0}\left[1+\frac{3.46 a}{V_{b}}\left(\frac{\varepsilon_{m}^{*}-1}{\varepsilon_{m}^{*}+2}\right)+\frac{15 a^{2}}{26 V_{b}} \frac{\rho q\left(\varepsilon_{m}^{*}-1\right) \sin ^{2} \theta_{0}}{6 \varepsilon_{0} E_{0}\left(1-\cos \theta_{0}\right)}\right]
$$

It noted that while $\mathrm{q}=0$ on eqn. (8), the corresponding propagation coefficient is

$$
K=k_{0}\left[1+\frac{3.46 a}{V_{b}}\left(\frac{\varepsilon_{m}^{*}-1}{\varepsilon_{m}^{*}+2}\right)\right]
$$

It is the same as the result of the propagation coefficient of Rayleigh approximation of sand particles without charge [3]. It can be concluded that the existing result of propagation coefficient of sand particles under the Rayleigh approximation is a special case of the result presented by this paper.

Substituted equation (8) into equations (2), (3), and the expression obtained for the attenuation and phase shift due to charged sand particles along horizontal paths in $(\mathrm{dB} / \mathrm{m})$ and phase shift $(\mathrm{deg} / \mathrm{m})$ are

$$
\alpha=8.686 k_{0}\left[-\frac{3.46 a}{V_{b}} \operatorname{Im}\left(\frac{\varepsilon_{m}^{*}-1}{\varepsilon_{m}^{*}+2}\right)+\frac{15 a^{2} \rho q \sin ^{2} \theta_{0}}{26 V_{b} \varepsilon_{0} E_{0}\left(1-\cos \theta_{0}\right)} \operatorname{Im}\left(\varepsilon_{m}^{*}-1\right)\right]
$$

$\beta=57.296 k_{0}\left[\frac{3.46 a}{V_{b}} \operatorname{Re}\left(\frac{\varepsilon_{m}^{*}-1}{\varepsilon_{m}^{*}+2}\right)+\frac{15 a^{2} \rho q \sin ^{2} \theta_{0}}{26 V_{b} \varepsilon_{0} E_{0}\left(1-\cos \theta_{0}\right)} \operatorname{Re}\left(\varepsilon_{m}^{*}-1\right)\right]$

\section{MicRowaVe Propagation ALONG EARTH-SPACE PATHS}

The average radii and visibility decrease with the height increase along earth-space paths [14]

$$
\begin{aligned}
a & =a_{0 a}\left(\frac{h}{h_{0}}\right)^{-\gamma_{a}}, \gamma_{a}=0.15 \\
V_{b} & =V_{b 0} \exp \left[b\left(h-h_{0}\right)\right], b=1.25
\end{aligned}
$$

Where $a$ and $a_{0 a}$ are radii at heights $h$ and $h_{0}$, respectively. The $V_{b}$ and $V_{b 0}$ are visibility at heights $h$ and $h_{0}$, respectively. The $h_{0}$ is height of earth station.
Substituting equations (12), (13) into equations (10), (11), the attenuation coefficient and phase shift coefficient at different height may be expressed as

$$
\begin{aligned}
\alpha=8.686 k_{0} & \frac{1}{\exp \left[b\left(h-h_{0}\right)\right]}\left[-\frac{3.46 a_{0 a}}{V_{b 0}}\left(\frac{h}{h_{0}}\right)^{-\gamma_{a}} \operatorname{Im}\left(\frac{\varepsilon_{m}^{*}-1}{\varepsilon_{m}^{*}+2}\right)\right. \\
& \left.+\frac{15 a_{0 a}{ }^{2} \rho q \sin ^{2} \theta_{0}}{26 V_{b 0} \varepsilon_{0} E_{0}\left(1-\cos \theta_{0}\right)}\left(\frac{h}{h_{0}}\right)^{-2 \gamma_{a}} \operatorname{Im}\left(\varepsilon_{m}^{*}-1\right)\right](14) \\
\beta=57.296 k_{0} & \frac{1}{\exp \left[b\left(h-h_{0}\right)\right]}\left[\frac{3.46 a_{0 a}}{V_{b 0}}\left(\frac{h}{h_{0}}\right)^{-\gamma_{a}} \operatorname{Re}\left(\frac{\varepsilon_{m}^{*}-1}{\varepsilon_{m}^{*}+2}\right)\right. \\
& \left.+\frac{15 a_{0 a}{ }^{2} \rho q \sin ^{2} \theta_{0}}{26 V_{b 0} \varepsilon_{0} E_{0}\left(1-\cos \theta_{0}\right)}\left(\frac{h}{h_{0}}\right)^{-2 \gamma_{a}} \operatorname{Re}\left(\varepsilon_{m}^{*}-1\right)\right]
\end{aligned}
$$

The attenuation and phase shift quantity along the earthspace paths can be expressed as:

$$
\begin{aligned}
& A_{m}=\int_{h 0}^{h_{m}} \alpha \cdot d h / \sin \theta \\
& \Phi_{m}=\int_{h_{0}}^{h_{m}} \beta \cdot d h / \sin \theta
\end{aligned}
$$

where $h_{m}$ is the height of sand storms.

\section{CAlculations and Results}

\section{A. Attenuation and Phase Shift along horizontal path}

In order to analyze the attenuation and phase shift of microwave induced by charged sand particles, the parameters are frequency $\mathrm{f}=37 \mathrm{GHz}$, dielectric constant $\varepsilon_{m}^{*}=4.0-j 1.3$ with moisture content $10 \%$, and $\mathrm{f}=14 \mathrm{GHz}, \varepsilon_{m}^{*}=3.9-j 0.62$ with moisture content $5 \%$ [3],[8], $E_{0}=0.5 \mathrm{~V} / \mathrm{m}, \mathrm{q}=-0.1 \mu \mathrm{C} / \mathrm{kg}$, $\theta_{0}=1.5 \mathrm{rad}$. Using eqns. (10) and (11), the relations attenuation and phase shift with visibility are shown in Fig.1 and Fig.2. As we can be seen, the attenuation and phase shift decrease with the increase of visibility, and the attenuation and phase shift for charged sand particles are larger than that for sand particles without charge. The results indicate that the larger frequency is, the larger the attenuation and phase shift are.

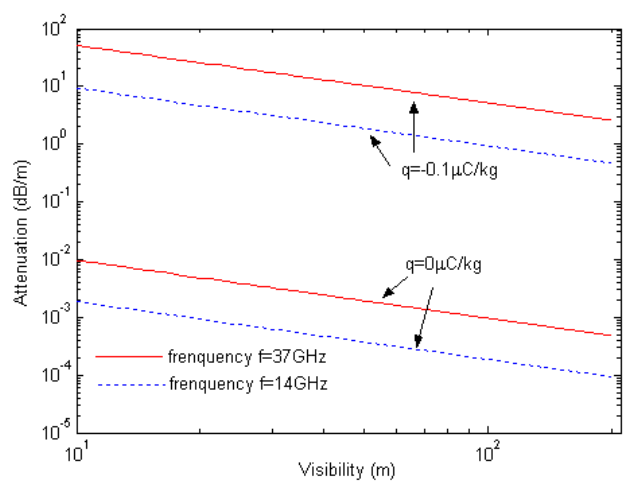

Fig. 1. Variation of attention with visibility 


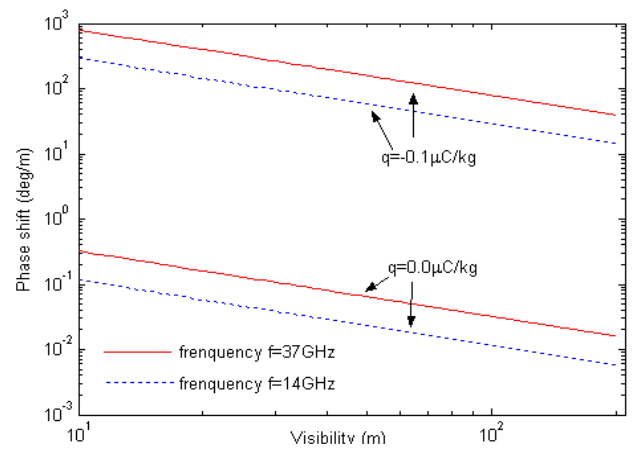

Fig. 2. Variation of phase shift with visibility

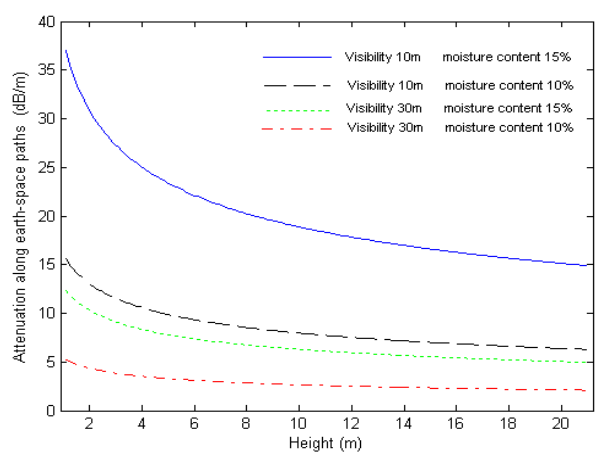

Fig. 3. Variation of attenuation with height along earth-space paths

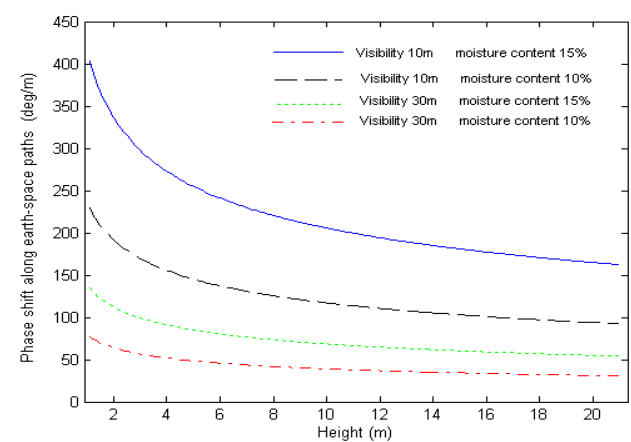

Fig.. 4. Variation of phase shift with height along earth-space paths

\section{B. Attenuation and Phase Shift along earth-space paths}

Using eqns. (14) and (15), the attenuation and phase shift are calculated for heights ranging from 1 to $21 \mathrm{~m}$. Using values of frequency $\mathrm{f}$, dielectric constant $\varepsilon_{m}^{*}$ and moisture content $\mathrm{p}$, namely $\mathrm{f}=37 \mathrm{GHz}, \quad \varepsilon_{m}^{*}=4.0-\mathrm{i} 1.3$ with $\mathrm{p}=10 \%$, $\varepsilon_{m}^{*}=6.72-\mathrm{i} 3.19$ with $\mathrm{p}=15 \%$ [15]. The charge-to-mass ratio is $q=-0.1 \mu \mathrm{C} / \mathrm{kg}$. The relations of attenuation and phase shift with height of storms are shown in Fig. 3 and Fig.4 with given visibility. It is clear from Fig. 3 that, for the same moisture content, attenuation decreases with the increase of height and visibility. For the same height, Fig. 3 shows that attenuation increases with the increase of moisture content.

From Fig.4, it shows that for the same moisture content, phase shift decreases with the increase of height and visibility. And for the same height, phase shift increases with the increase of moisture content, and phase shift decreases with the increase of visibility.

\section{CONCLUSIONS}

This paper discusses the propagation characteristics of microwave wave through sand storms incorporating charged sand particles. The result shows that attenuation and phase shift decrease with the increase of visibility, when the electric charges distribute on partial surface of the spherical sand particles, the attenuation with charged sand is obviously larger than without regard to charges on sand. Because of the electric charges on the sand surface, the attenuation becomes increased. Based on the analysis of microwave waves propagating along horizontal paths, expressions of attenuation and phase due to sand storms on earth-space paths are derived and calculated by using typical data.

\section{ACKNOWLEDGMENT}

This works is supported by the National Natural Science Foundation of China (Grant No. 61102018, 61271110, 61073106)

\section{REFERENCES}

[1] T.S. chu, "Effect of sand storms on microwave propagation," Bell Syst. Tech.J., Vol.58, No.2, 549-555, 1979.

[2] A. O. Bashir, N. J. Mcewan, "Microwave propagation in dust storms: A review." IEEE Proceedings H, 1986, 133(3), 241-247, 1986.

[3] A.J. Ansari and B.G.,Evans, "Microwave propagation in sand and dust storms," IEE Proc. F, Commun., Radar \& Signal Process., ,vol.129, pp. 315-322,1982.

[4] S. I. Ghobrial, "The effect of sand storms on microwave propagation," Proc. Nat. Telecommun. Conf., Vol. 2, Proc.43.5.1-43.5.4, Houston, TX, 1980.

[5] B. R. Vishvakarma, and C. S. Rai, "Limitations of Rayleigh scattering in the prediction of millimeter wave attenuation in sand and dust storms," Geoscience and Remote Sensing Symposium, IEEE Inter., 1993.

[6] Z. Elabdin and M. R. Islam, "mathematical model for the prediction of microwave signal attenuation due to duststorm," Progress In Electromagnetics Research M, Vol. 6, 139-153, 2009.

[7] Y. Wenyan and W. wei, "Effect of dust storm on the ear-space microwave and millimeter wave propagation," Journal of northwestern polytechnical university, Vol.9, No.4, 484$492,1991$. 
[8] X. Ying Xia and H. Jiying, "Effect of sand and dust storms on Ka-band electromagnetic wave propagation along earth-space paths." Chinese Journal of Radio Science, vol.18, 328-331, 2003.

[9] Y. Ruike, W. Zhensen, and Y. Jinguang, "The study of MMW and MW attenuation considering multiple scattering effect in sand and dust storms at slant paths," International Journal of Infrared and Millimeter Waves, Vol. 24, No. 8,pp.1383-1392, 2003.

[10] E. Norinpel, "Microwave attenuation due to dust and sand storm in earth-satellite link," Strategic Technology, International Forum, pp. 599- 601, 2007.

[11] Z. Xiaojing, H. Ning, "Laboratory measurement of electrification of wind-blown sands and simulation of its effect on sandsaltation movement." Journal of Geophysical Research, vol.108, pp. 43-22, 2003.
[12] H. Shuqin, Z. Youhe, "Scattering field of charged sand particles and influence of propagation characteristic of electromagnetic wave." Science in China, vol.35, pp. 308-317, 2005.

[13] D. Qunfeng, J.-D. Xu. et al, “Attenuation effects of microwave through charged sand particles," High Power Laser and Particle Beams,Vol.21,No.10,pp. 1517-1520, 2009.

[14] A.S. Ahmed, A.A. Ali and M.A. Alhaider, "Airborne Dust Size Analysis for Tropospheric Propagation of Millimetric Waves into Dust Storms," IEEE Transactions on Geoscience and Romote Sensing, Vol.25, No.5,pp.593-599,1987.

[15] L. Yingle. and P. Yang, "The permittivity based on electromagnetic wave attenuation for rain medium and its applications," Journal of Electromagnetic Waves and Applications, Vol. 20, No. 15, 2231-2238, 2006. 